\title{
Are Aerobics Trophic for Cognition in Late Life?
}

\author{
Jeff D. Sanders • Dilip V. Jeste
}

Published online: 28 January 2011

(C) The Author(s) 2011. This article is published with open access at Springerlink.com

\section{Trial}

Baker LD, Frank LL, Foster-Schubert K, et al.: Effects of aerobic exercise on mild cognitive impairment: a controlled trial. Arch Neurol 2010, 67:71-79.

Rating: •• Of major importance.

Introduction: Aging of the population is an issue of great public health significance. Forecasts indicate that the number of Americans 65 years of age and older will increase from 39 million in 2008 to between 99 million and 108 million by 2050 [1]. The population aged 85 years or older is projected to rise from 5.4 million in 2008 to between 25 million and 35 million by 2050 [1]. These changes will bring new challenges in treating a greater number of older adults with psychiatric conditions [2]. Especially important will be the recognition, prevention, and treatment of neurocognitive disorders. Cognitive ability is a significant predictor of self-rated successful aging [3]. Indeed, cognitive ability is one of the major correlates of successful aging, along with optimism, resilience, and physical and mental health-related quality of life [3].

Age-associated cognitive decline that is greater than expected but without significant functional disability is designated mild cognitive impairment (MCI). MCI is an increasingly recognized neuropsychiatric problem in the aging population that is an important risk factor for

\footnotetext{
J. D. Sanders $\cdot$ D. V. Jeste $(\bowtie)$

Sam and Rose Stein Institute for Research on Aging,

University of California, San Diego,

9500 Gilman Drive \#0664,

San Diego, CA 92093, USA

e-mail: djeste@ucsd.edu
}

dementia and subsequent institutionalization [4-6]. Despite these data, no medications are currently available for treating MCI in the aging population that are approved by the US Food and Drug Administration [6].

It is reasonable to hypothesize that exercise may be an important intervention for promoting successful cognitive aging. Exercise has a documented role in preventing cellular and molecular changes related to aging [7, 8]. Furthermore, exercise has been shown to improve at least some aspects of cognition in animal models and in clinical investigations [9, 10]. Data from the present study suggest that aerobic exercise may be an effective nonpharmacologic intervention for preserving healthy cognition and possibly alleviating disability associated with MCI in late life.

Aims: This study examined the effects of aerobic exercise on cognition in sedentary older men and women with MCI. The authors investigated the effects of prolonged highintensity aerobic exercise on multiple neuropsychological measures of cognition. The study also examined the effects of aerobic exercise on metabolic indices and on putative biomarkers of Alzheimer's disease. The authors further investigated the role of gender in predicting outcome.

Methods: The investigators performed an extensive screening of 466 adults by telephone. Of the adults screened, 426 were excluded for a variety of reasons, such as declining participation, not having a history of memory impairment, not being sedentary, or having a medical condition that precluded their participation (ie, unstable cardiac disease, cerebrovascular disease, musculoskeletal impairment, or other conditions with significant medical sequelae). Seven individuals were excluded because they withdrew consent, failed treadmill tests, or experienced vasovagal syncope with blood draw. 
At enrollment, 33 sedentary adults between the ages of 55 and 85 who were diagnosed with MCI were randomly assigned to aerobic exercise or a control group. MCI was diagnosed using Petersen criteria [11]. Participants were allowed to continue taking $\beta$-blockers or statins but not allowed to take antidiabetes medications. The exercise intervention consisted of an exercise routine 4 day/week for $45-60 \mathrm{~min} / \mathrm{session}$. Exercise involved a regimen of gradually increasing intensity until participants achieved $75-85 \%$ of their heart rate reserve.

Cognitive tests, metabolic tests, and biomarker assays were performed at baseline, 3 months, and 6 months. Cognitive tests included Symbol Digit Modalities, Verbal Fluency, Stroop, Trails B, Task Switching, Story Recall, and List Learning. Metabolic parameters included total body fat, body mass index, cholesterol, lipids, triglycerides, insulin sensitivity, and cortisol. Biomarkers included brainderived neurotrophic factor (BDNF) and $\beta$-amyloid protein. Four individuals (two men and two women) in the aerobic exercise group discontinued the study before completing 6 months of intervention. These individuals were noted to have higher fasting baseline plasma glucose levels than completers but were similar in other baseline measurements. The primary analysis included 10 women and 9 men in the exercise group and 5 women and 5 men in the stretching control group.

Results: At enrollment, the exercise and control groups were similar in mean age, cognitive performance, physical fitness, and metabolic parameters. At baseline, women were noted to have higher Mini-Mental State Examination (MMSE) scores, body fat, cholesterol, and cortisol levels, and lower insulin-like growth factor 1 levels than men. Six months of high-intensity exercise improved exercise treadmill test assessment of $\mathrm{VO}_{2}$ peak, a measure of maximum volume of oxygen consumption that reflects physical fitness. This exercise regimen also improved treadmill grade and treadmill time to exhaustion relative to stretching control. Neither gender nor $\beta$-blocker use was found to affect these findings.

After 6 months of high-intensity exercise, a significant improvement was observed in executive functioning skills of multitasking, cognitive flexibility, information-processing efficiency, and selective attention. Further analysis revealed gender-specific effects on cognition, cortisol, and BDNF. In women, increasing $\mathrm{VO}_{2}$ peak was associated with improved executive functioning. Also, in women, high-intensity exercise resulted in a statistically significant improvement on Symbol Digit Modalities, Verbal Fluency, and Stroop tests, and a trend toward improvement on Trails B. Analysis of these measured at 3 months did not reach statistical significance, indicating that a prolonged duration of exercise may be necessary to attain these benefits.
Cognitive improvements were paralleled by statistically significant increases in insulin sensitivity and decreases in plasma cortisol and BDNF. In men, high-intensity exercise resulted in a trend toward improvement on Trails B alone and a statistically significant increase in plasma cortisol and BDNF. Men and women demonstrated similar improvements in cardiac fitness and similar decreases in body fat, cholesterol, and low-density lipoproteins.

Discussion: The authors concluded that aerobic exercise is an important intervention that may mitigate $\mathrm{MCI}$ in older individuals. The improved performance by women across multiple neuropsychological domains, as contrasted with a limited response by men, suggests that aging women may find aerobic exercise especially beneficial to cognitive function. The cognitive improvements in women may be related to exercise-induced alterations in the hypothalamicpituitary-adrenal axis, glucose metabolism, and BDNF that are not seen in men.

\section{Comments}

This study had several notable strengths. It used extensive screening to ensure that the participants qualified as sedentary older adults with MCI who were physically fit enough to meet the demands of intensive exercise. These individuals were then randomly assigned to an aerobic exercise group or a stretching control group. The exercise intervention was a well-defined regimen consisting of an exercise routine 4 day/week for $45-60 \mathrm{~min} / \mathrm{session}$ for 6 months. The intensity of the exercise routine was monitored so that participants were exercising at $75-80 \%$ of heart rate reserve. A reasonably detailed assessment of neuropsychological functioning and accompanying physiologic assessments and biomarker assays were performed at the study's completion.

However, these encouraging results should be considered in the context of this study's limitations. First, it is not clear how the original 466 adults who were screened were selected. Information is not provided on what guided the investigators to contact these individuals and from what population they were contacted. Furthermore, in their initial screening of 466 adults, 113 were excluded because of a significant medical condition. Therefore, these findings may have limited generalizability to real world older adult populations, although this is understandable in view of the nature of the intervention (ie, high-intensity aerobic exercise for 6 months). The primary analysis was based on a small sample of 19 individuals in the aerobic group and 10 in the control group. Future studies with a lower intensity exercise regimen may allow for less strict enrollment screening and allow for a greater sample number and greater applicability. 
Although the study collected considerable data at baseline, an even greater detail of baseline data would have allowed for a more rigorous analysis of treatment and control group equivalence at enrollment. For instance, although data are provided on the number of participants on $\beta$-blockers and statins, it is not known which other medications the individuals were taking during the study that might have impacted on their cognitive performance. Although the authors screened and excluded patients with significant medical sequelae, information on the number of less severe conditions affecting each treatment group is not provided. Individuals within this age range may be affected by many medical conditions that did not warrant exclusion but whose presentation or treatment may have an effect on cognitive performance $[12,13]$.

A greater sample number that included additional baseline data analysis would also allow for a more thorough assessment of the gender specificity of the results reported. For example, while women in the aerobic exercise group displayed a selective improvement on many neuropsychological tests, at enrollment, they were also noted to have higher MMSE scores, body fat, cholesterol, and cortisol levels, and lower insulin-like growth factor 1 levels than men. It would be interesting to examine whether men with comparable baseline MMSE or metabolic profiles, relative to women counterparts, would demonstrate similar improvements in cognition with high-intensity aerobic exercise. These may be important variables that predict cognitive improvement with aerobic exercise in aging populations that are independent of gender.

Future studies examining the neurobiological substrates for the cognitive-enhancing effects of exercise will also be an exciting new direction. Recent findings have implicated sestrin and target-of-rapamycin signaling as important mechanisms in age-related neuropathologies [14]. To date, few investigations have addressed how exercise may affect these signaling pathways in the aging brain. Studies addressing the impact of exercise on these signaling pathways could provide important basic scientific data to complement the clinical inquiries.

In summary, this is an important study that provides groundwork for more extensive research. Given the potential disability associated with cognitive impairment in older people and limited data on effective interventions, it provides important preliminary information on the effects of exercise in MCI.

\section{Disclosure}

No potential conflicts of interest relevant to this article were reported.

Open Access This article is distributed under the terms of the Creative Commons Attribution Noncommercial License which permits any noncommercial use, distribution, and reproduction in any medium, provided the original author(s) and source are credited.

\section{References}

1. Olshanksy SJ, Golman DP, Zheng Y, et al.: Aging in America in the twenty-first century: demographic forecasts from the MacArthur Foundation Research Network on Aging Society. Milbank Q 2009, 87:622-842.

2. Jeste DV, Alexopoulos GS, Bartels SJ, et al.: Consensus statement on the upcoming crisis in geriatric mental health: research agenda for the next two decades. Arch Gen Psychiatry 1999, 56:848-853.

3. Jeste DV, Depp CA, Vahia IV: Successful cognitive and emotional aging. World Psychiatry 2010, 9:78-84.

4. Luck T, Luppa M, Briel S, et al.: Mild cognitive impairment: incidence and risk factors: results of the Leipzig longitudinal study of the aged. J Am Geriatr Soc 2010, 58:1903-1910.

5. Boyle PA, Wilson RS, Aggarwal NT, et al.: Mild cognitive impairment: risk of Alzheimer disease and rate of cognitive decline. Neurology 2006, 67:441-445.

6. Roberts JS, Karlawish JH, Uhlmann WR, et al.: Mild cognitive impairment in clinical care: a survey of American Academy of Neurology members. Neurology 2010, 75:425-431.

7. Puterman E, Lin J, Blackburn E, et al.: The power of exercise: buffering the effect of chronic stress on telomere length. PLoS ONE 2010, 5:e10837.

8. Valdez G, Tapia JC, Kang H, et al.: Attenuation of age-related changes in mouse neuromuscular synapses by caloric restriction and exercise. Proc Natl Acad Sci U S A 2010, 107:14863-14868.

9. Gobeske KT, Das S, Bonaguidi MA, et al.: BMP signaling mediates effects of exercise on hippocampal neurogenesis and cognition in mice. PLoS ONE 2009, 4:e7506.

10. Yangisawa H, Dan I, Tsuzuki D, et al.: Acute moderate exercise elicits increases dorsolateral prefrontal activation and improves cognitive performance with Stroop test. Neuroimage 2010, 50:1702-1710.

11. Petersen RC: Mild cognitive impairment as a diagnostic entity. J Intern Med 2004, 256:183-194.

12. Wagg A, Verdejo C, Molander U: Review of cognitive impairment with antimuscarinic agents in elderly patients with overactive bladder. Int J Clin Pract 2010, 64:1279-1286.

13. Jae KS, Hie LJ, Young LD, et al.: Neurocognitive dysfunction associated with sleep quality and sleep apnea in patients with mild cognitive impairment. Am J Geriatr Psychiatry 2010 (Epub ahead of print).

14. Lee JH, Budanov AV, Park EJ, et al.: Sestrin as a feedback inhibitor of TOR that prevents age-related pathologies. Science 2010, 327:1223-1228. 